\title{
AU QUÉBEC, MIEUX S'INFORMER POUR MIEUX PROTÉGER
}

À l'instar d'autres entreprises, les organismes québécois de protection des forêts contre le feu subissent des pressions reliées à l'augmentation des coûts de contrôle des incendies et à leurs propres restrictions budgétaires. Au même moment, les "clients" du système désirent que la qualité du service soit au moins maintenue, sinon améliorée. Ainsi en est-il du public, utilisateur des espaces forestiers ou résidant à proximité de la forêt, qui aime se sentir à l'abri des catastrophes dues aux incendies forestiers. Autre élément crucial: l'industrie et les gouvernements investissent chaque année des centaines de millions de dollars dans l'aménagement des forêts.

Au Québec, la dernière révision des structures pour la protection contre l'incendie remonte à 1972. L'adoption de nouveaux équipements et, plus récemment, les développements du côté des technologies de l'information permettent de considérer de nouvelles approches pour faire face aux contraintes et pour améliorer l'efficacité. Parmi celles-ci, il y a le système centralisé de prise de décisions. Sous un tel système quelques personnes ont la responsabilité de la détection et de la suppression des feux à l'intérieur d'une vaste région. Ce groupe doit pouvoir compter sur une technologie permettant la collecte et l'analyse rapide des données.

Le présent article vise à faire le point sur le développement et les applications d'un système d'information organisationnel pour la protection des forêts contre le feu au Québec. Mais d'abord, l'auteur discute du contexte dans lequel il s'intègre.

\section{LE CONTEXTE}

Les statistiques et l'expérience nous enseignent que "l'homme" demeure inefficace dans le combat des grands incendies forestiers. Peu de feux d'envergure ont pu être maîtrisés par la seule intervention humaine, en dépit des sommes importantes et des efforts investis dans le combat. Ces incendies s'éteignent généralement d'eux-mêmes, faute de combustible disponible ou suite à un changement marqué des conditions météorologiques. Les gestionnaires de la ressource forestière doivent être prêts à accepter un certain niveau de pertes dues aux feux d'envergure, à coup sûr les plus significatifs quant aux dommages qu'ils causent. L'amélioration de l'efficacité des systèmes de protection contre l'incendie passe par la réduction du nombre de feux d'envergure. Au Québec, les responsables de la protection des forêts reconnaissent la justesse de ce constat. L'objectif fondamental des organismes québécois de protection en fait foi: prévenir les incendies forestiers par une action soutenue de sensibilisation et détecter rapidement les débuts de feux de façon à pouvoir intervenir le plus tôt possible alors qu'ils sont de dimension réduite. 
Depuis plusieurs années, la poursuite de cet objectif avec des moyens de plus en plus modernes et efficaces a permis d'améliorer les résultats. Le tableau I ci-dessous indique en effet que, depuis 1972, la superficie moyenne des feux au Québec a diminué de moitié par rapport à la période équivalente précédente. La superficie moyenne détruite annuellement s'élève à 36000 hectares, soit un sixième des 225000 hectares récoltés en forêt publique. Ces statistiques s'appliquent au territoire sous protection intensive, soit quelque $523000 \mathrm{~km}^{2}$ de boisés commercialement exploitables. Le coût de l'ensemble du programme atteint annuellement 34 millions de dollars (dollar canadien de 1989). Une importante partie du budget est allouée à la prévention: les données du tableaull ci-dessous montre que $85 \%$ des sommes servent à défrayer les frais de fonctionnement récurrents du système (incluant les frais fixes d'opérations des avions-citernes) alors que les autres $15 \%$ sont encourus au moment de la lutte proprement dite.

Comme on doit s'y attendre, il y a des variations importantes d'une année à l'autre dans le nombre de feux, leurs causes et les superficies affectées. Le tableau III ci-dessous nous permet

Tableau I Incendies forestiers au Québec

Moyenne selon les périodes

\begin{tabular}{|c|c|c|c|}
\hline Périodes & $\begin{array}{c}\text { Nombre de feux } \\
\text { par année }\end{array}$ & $\begin{array}{c}\text { Superficie affectée } \\
\text { annuellement (ha) }\end{array}$ & $\begin{array}{c}\text { Superficie des feux } \\
\text { (ha/feu) }\end{array}$ \\
\hline 1956 à $1971 \ldots \ldots \ldots \ldots \ldots \ldots$ & 885 & 61835 & 70 \\
1972 à $1988 \ldots \ldots \ldots \ldots \ldots \ldots$ & 1057 & 34531 & 33 \\
\hline
\end{tabular}

Tableau II Coût de la protection des forêts contre le feu au Québec en 1989-1990

\begin{tabular}{|c|c|c|}
\hline Élément de coût & $\begin{array}{l}\text { Dépenses } \\
(000000 \$)\end{array}$ & $\begin{array}{l}\% \text { par rapport } \\
\text { au coût total }\end{array}$ \\
\hline Coût de prévention & 29,0 & 85 \\
\hline Coût d'extinction & 5,0 & 15 \\
\hline TOTAL & 34,0 & 100 \\
\hline
\end{tabular}

Tableau III

Incendies forestiers au Québec

Années les plus désastreuses entre 1941 et 1987

\begin{tabular}{|c|c|c|c|c|c|c|}
\hline Années & Nombre & $\begin{array}{l}\text { Superficie } \\
\text { totale (ha) }\end{array}$ & \multicolumn{4}{|c|}{ Feux de foudre } \\
\hline & & & Nombre & (\%) & Sup. (ha) & $(\%)$ \\
\hline $\begin{array}{l}1941 \ldots \ldots \ldots \\
1944 \ldots \ldots \ldots\end{array}$ & $\begin{array}{l}1710 \\
1542\end{array}$ & $\begin{array}{l}642455 \\
242326\end{array}$ & $\begin{array}{l}102 \\
169\end{array}$ & $\begin{array}{r}6 \\
11\end{array}$ & $\begin{array}{r}65302 \\
4989\end{array}$ & $\begin{array}{r}10 \\
2\end{array}$ \\
\hline $\begin{array}{l}1951 \ldots \ldots \ldots \\
1953 \ldots \ldots \ldots \\
1955 \ldots \ldots \ldots\end{array}$ & $\begin{array}{r}869 \\
2257 \\
1276\end{array}$ & $\begin{array}{l}108134 \\
232090 \\
222018\end{array}$ & $\begin{array}{r}44 \\
381 \\
304\end{array}$ & $\begin{array}{r}3 \\
17 \\
24\end{array}$ & $\begin{array}{r}2660 \\
63386 \\
93795\end{array}$ & $\begin{array}{r}2 \\
27 \\
42\end{array}$ \\
\hline $\begin{array}{l}1962 \ldots \ldots \ldots \\
1967 \ldots \ldots \ldots \\
1968 \ldots \ldots \ldots \ldots\end{array}$ & $\begin{array}{r}1249 \\
939 \\
1164\end{array}$ & $\begin{array}{l}199185 \\
108751 \\
131114\end{array}$ & $\begin{array}{l}301 \\
251 \\
235\end{array}$ & $\begin{array}{l}24 \\
27 \\
20\end{array}$ & $\begin{array}{l}59202 \\
82048 \\
90199\end{array}$ & $\begin{array}{l}30 \\
75 \\
69\end{array}$ \\
\hline $1971 \ldots$ & 1331 & 261650 & 340 & 26 & 105428 & 40 \\
\hline $\begin{array}{l}1983 \ldots \ldots \ldots \ldots \\
1986 \ldots \ldots \ldots \ldots\end{array}$ & $\begin{array}{r}1653 \\
830\end{array}$ & $\begin{array}{l}238903 \\
167550\end{array}$ & $\begin{array}{r}399 \\
93\end{array}$ & $\begin{array}{l}24 \\
11\end{array}$ & $\begin{array}{l}234447 \\
165563\end{array}$ & $\begin{array}{l}98 \\
99\end{array}$ \\
\hline
\end{tabular}

Les données de ce tableau concernent le territoire protégé tel que défini aux différentes époques. 
d'examiner les années les plus désastreuses depuis 1941. Avant 1953, le nombre de feux et les vastes superficies détruites étaient surtout attribuables à d'autres causes que la foudre. Cela tend à démontrer que les efforts de prévention ont porté fruits et qu'aujourd'hui, l'organisation contrôle plus efficacement les feux de causes humaines. Par ailleurs, la prédominance des feux de foudre, en terme de superficie dévastée, croît pour atteindre des maximums en 1983 et 1986. Ainsi, même s'ils sont moins nombreux (environ $25 \%$ du total), les feux de foudre sont devenus les plus dévastateurs. Leur maîtrise est fonction de leur fréquence, de leur potentiel, de la difficulté d'accès, de leur éloignement et surtout de leur simultanéité.

Le contrôle d'une situation de feux multiples pose, aux responsables de la protection des forêts, des problèmes de gestion de leurs ressources d'intervention. II faut parfois faire face à plusieurs feux en une période de quelques heures. Le 25 juillet 1988 par exemple, la foudre a allumé en un seul après-midi 78 incendies dans un secteur du Québec couvrant $200 \mathrm{~km}$ sur $400 \mathrm{~km}$. En pareil cas, il est nécessaire d'intensifier la détection aux moments propices et d'accroître la capacité d'attaque initiale. Pour ce faire, l'organisation de protection doit être en mesure de prévoir les situations potentiellement difficiles et posséder la flexibilité et la mobilité lui permettant de diriger effectifs et materiels vers les zones à risque élevé de début d'incendies forestiers et vers les feux prioritaires. Le système centralisé de gestion des incendies facilite grandement la concrétisation de ces deux caractéristiques.

L'organisation actuelle de la protection contre le feu au Québec est le fruit d'une longue évolution. Comme les incendies forestiers transgressent les frontières administratives et les juridictions politiques, la responsabilité de la protection des forêts revient au Gouvernement, par le biais du ministère de l'Énergie et des Ressources. Les responsables ont cependant voulu s'assurer de la collaboration du public, des utilisateurs forestiers ainsi que de tous ceux qui bénéficient de la protection des forêts. Au Québec, sept organismes régionaux de protection (les Sociétés de Conservation), à caractère unique au Canada, permettent depuis 18 ans aux divers intervenants de participer à la gestion de la protection contre le feu et ce, grâce à une participation au conseil d'administration de ces organismes et à la formule de financement des opérations. C'est par le biais de ces organismes que s'établit le lien essentiel entre les "clients" et l'État (Gouvernement du Québec), fournisseur du service de protection, en vue de l'intégration de la gestion des incendies à l'ensemble de l'amenagement forestier et du territoire.

Lorsque survient un feu, le niveau de réponse devant être exercé va de l'observation pure et simple dans les forêts nordiques jusqu'à l'attaque agressive dans les forêts commerciales et péri-urbaines. Cela requiert une communication efficace et constante entre les coopérateurs majeurs: le ministère de l'Énergie et des Ressources, les autres ministères et organismes gouvernementaux, l'industrie forestière et les municipalités. II est reconnu que les Sociétés de Conservation s'acquittent de cette tâche à la satisfaction de leurs membres (ministère de l'Énergie et des Ressources du Québec, bénéficiaires de contrat d'approvisionnement et d'aménagement forestier, grands propriétaires privés). Cette formule, qui repose sur un niveau poussé de délégation, est efficace et rien ne justifie une modification dans un proche avenir.

Au plan operationnel cependant, les technologies favorisent la centralisation de l'information et des décisions. Cela entraîne la révision périodique des structures opérationnelles. Au Québec, il est intéressant de constater que le profane trouve régulièrement des similitudes entre les opérations de détection et de combat des incendies forestiers et les opérations de services d'urgence tels la protection civile, les services d'incendies des grandes villes, les organisations militaires modernes, etc... II n'est donc pas surprenant de retrouver en protection des forêts une structure québécoise qui permet d'appliquer le système centralisé de gestion, lequel requiert:

- Le commandement et le contrôle d'heure en heure, exercés par le responsable de la performance au niveau d'une Société régionale de Conservation. Cette personne dirige le quartier général de lutte (QGL), véritable centre nerveux du combat. L'officier du QGL doit être en mesure d'évaluer les situations et de décider rapidement des stratégies. L'ampleur de l'organisation sur le terrain fluctue selon les situations: chaque personne répond à un supérieur et au sommet hiérarchique se retrouve l'officier du QGL. II porte évidemment la responsabilité de ses décisions.

- Des communications à la fine pointe de la technologie pour permettre la collecte et la synthèse des données, la diffusion des décisions jusqu'aux postes avancés et le retour des résultats vers les centres décisionnels. 
- L'information constamment renouvelée et mise à la disposition du décideur.

- Des ressources mobiles et en quantité suttisante pour détecter et combattre des feux. Un Centre de Coordination nationale de la Lutte $(C C L)$ dont le personnel est chargé de surveiller le déroulement des activités sur tout le territoire québécois et de déployer les ressources en fonction des besoins régionaux et des priorités. Au Québec, le CCL répartit notamment la flotte de 21 avions-citernes entre les différentes bases d'opérations et coordonne le transfert des autres ressources spécialisées, tels les équipes de choc et les aéropointeurs entre les différentes Sociétés régionales de Conservation.

Le succès du système centralisé dépend de la qualité de l'information mise à la disposition des personnes ceuvrant dans les QGL et au CCL. L'organisation doit donc pouvoir compter sur un système informatique destiné à la collecte des données et à la présentation des synthèses de l'information la plus récente sur les variations affectant 'l'environnement et les incendies forestiers. Cette composante porte l'appellation: Système de Gestion en Protection des Forêts (SGPF).

L'ordinateur y joue un rôle de premier plan. II est jumelé aux détecteurs de diverses natures: stations météorologiques, détecteurs d'éclairs, radars pour la mesure de la pluie, etc...

Matériels et logiciels assurent la collecte, l'archivage et la synthèse des données requises pour assister l'officier en devoir dans ses décisions journalières. En aucune façon, il n'est prévu que ces systèmes remplaceront le décideur. On compte plutôt que les résultats informatiques serviront à accroître la qualité des décisions et la confiance du décideur.

\section{LE SYSTÈME DE GESTION EN PROTECTION DES FORÊTS (SGPF)}

Les progrès réalisés dans trois techniques de pointe, à savoir l'ordinateur, la télédétection et les sciences de la gestion, ont abouti à la mise en place de systèmes très perfectionnés de gestion informatisée des incendies qui offrent la possibilité d'un renouveau total dans les opérations d'interventions. Ces systèmes visent d'abord l'identification des problèmes mais il est aussi prévu qu'ils pourront un jour suggérer des scénarios de solutions. L'objectif ultime du SGPF est de prévoir à l'avance les incendies forestiers, leur impact, et le résultat de l'application de diverses stratégies d'intervention.

Au Québec, six indices de danger d'incendie de torèts constituent autant d'outils pour élaborer une bonne stratégie de lutte. Le Systeme de Gestion en Protection des Forêts permet d'obtenir ces indices sous formes de graphiques où l'intensité des dangers de feu est représentée par différentes couieurs et de façon numérique: plus le chiffre (l'indice numérique) est élevé, plus il y a danger d'incendie. L'indice de combustible léger porte sur la nature de la litière présente en sous-bois ainsi que sur la pluie, l'humidité relative, la vitesse du vent et la température. L'indice de l'humus s'intéresse à la couche de matière organique en décomposition sous la litière. L'indice de sécheresse tient compte des combustibles présents en profondeur ainsi que de la pluie et de la tempèrature. L'indice de propagation initiale varie surtout en fonction de la vitesse du vent. L'indice du combustible disponible combine les informations relatives aux peuplements forestiers, à la sécheresse et à l'humus. Enfin l'indice Forêt-Météo synthétise l'ensemble des indices.

La prévision de l'impact des feux est à la fois fonction de la valeur économique de la forêt menacée et des conséquences des incendies au plan écologique. Les connaissances dans ce domaine sont présentement limitées. Néanmoins, la prévision fiable de la propagation et de l'impact d'un feu pourrait logiquement amener le décideur à la non-intervention dans certains cas, s'il était estimé que les effets bénéfiques du feu l'emporteraient sur ses conséquences négatives.

La prévision des résultats des stratégies d'intervention requiert les inventaires des ressources de lutte disponibles (effectifs, matériels, aéronefs), la connaissance de leurs taux de productivité dans diverses conditions et la capacité de simuler différents niveaux d'attaque selon des combinaisons variées de ressources. Peu de travaux ont été entrepris à ce jour pour cerner cet aspect bien que les tentatives récentes, basées sur le développement de "systèmes experts " à intelligence artificielle, semblent très prometteuses. 
Figure 1 STRUCTURE GÉNÉRALE DU SYSTÈME DE GESTION EN PROTECTION DES FORÊTS DU QUÉBEC (SGPF) *

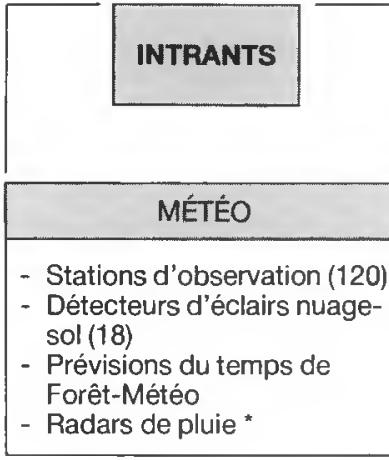

\section{INCENDIES FORESTIERS}

- Rapport initial

- Rapport final (126 champs)

- Sources de chaleur rapportées par les capteurs infrarouge *

- Taux de propagation des feux *

\section{BASES DE DONNÉES ET LOGICIELS}

PRODUITS

\section{BASES DE DONNÉES}

1 - Météorologie (10 ans)

2 - Feux (10 ans)

3 - Foudre (année en cours)

4 - Géographie du territoire *

5 - Inventaires (ressources *)

SYSTĖME DE GESTION

DES BASES DE DONNÉES

LOGICIELS

- Communications (QNET)

- Systèmes de surveillance des opérations du SGPF

- Calcul d'indices

- Prévision des feux

- Affichage graphique (cartes graphiques à l'écran et reproduction sur traceuse)

- Modèles d'optimisation *

- Intelligence artificielle (MPRO LOG) *
- Combustibles forestiers *

- Relief*

- Impact du feu (valeur économique de la forêt et effet écologique du feu) *

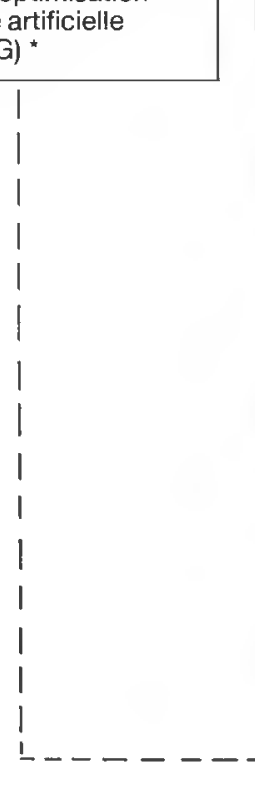

\section{MÉTÉO}

- Classes de température, humidité relative et pluie

- Carte du temps observé et prévu

- Indices Forêt-Météo observés et prévus

\begin{tabular}{|c|}
\hline FOUDRE \\
\hline $\begin{array}{l}\text { - Affichage des points de } \\
\text { chute des éclairs }\end{array}$ \\
\hline
\end{tabular}

\section{FEUX}

- Localisation des feux actifs et éteints

- Prévision des feux de causes humaines

- Prévision des feux de foudre

- Modèle de propagation des feux *

- Brûlage dirigé *

\section{PATROUILLES AÉRIENNES}

- Fréquence *

- Routes *

- Nombre d'appareils *

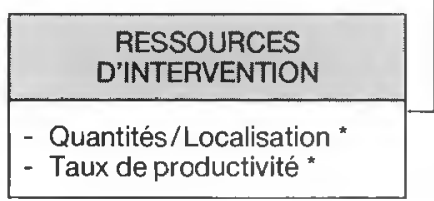

* Eléments au stade du développement et de l'experimentation. 
En se basant sur cette vue d'ensemble du SGPF et en reconnaissant les potentiels qu'il recèle, les responsables de la protection au Québec ont décidé de l'appliquer à l'organisation. Pour ce faire, le Québec, avec l'aide de Forêts-Canada, a mis sur pied le Centre de Transfert technologique de Maniwaki (CTTM). Son principal mandat consiste à faciliter et à accélérer le transfert des technologies développées par l'Institut forestier national de Petawawa (IFNP) aux Sociétés de Conservation et au Centre de Coordination de la Lutte du Québec. Le CTTM offre en outre ses services et son expertise ailleurs au Canada et dans le monde.

La figure 1 (p. 350) illustre la structure générale du SGPF. II convient de noter que plusieurs éléments du Système sont encore au stade du développement et de l'expérimentation (ils sont identifiés par un astérisque sur la figure 1). Les travaux de recherche et de développement dans ce domaine sont conduits par l'IFNP. Le Québec a toujours soutenu ces activités.

Au plan des équipements, le Québec opère un réseau de détecteurs de foudre comprenant 18 stations de repérage, dont trois couvrent le territoire de la Baie James. Ces appareils effectuent la localisation des éclairs s'abattant au sol et identifient certaines de leurs caractéristiques. En outre, les Sociétés de Conservation possèdent des ordinateurs de marque Vax. Ces appareils sont reliés à l'ordinateur central du Centre de Coordination de la Lutte à Québec par le biais du logiciel de communication appelé QNET.

Développé par le CTTM, le logiciel QNET fournit un moyen de transférer, de façon efficace et à un coût réduit, les données entre le $\mathrm{CCL}$ et les QGL des régions. Ainsi les différents ordinateurs s'échangent, suivant en cela un horaire quotidien pré-établi, les données relatives aux incendies et à la météorologie qui sont saisies localement. C'est à partir de ces informations que sont ensuite produits les graphiques montrant les indices de danger de feu et les situations prévisibles à travers la province. L'ordinateur central est relié à Forêt-Météo du Service canadien de l'Environnement atmosphérique, à Montréal. Cette portion du réseau permet la redistribution des prévisions du temps et des autres produits de Forêt-Météo. La figure 2 ci-dessous montre le réseau de communications informatisées en protection des forêts.

Outre le logiciel de communication, le SGPF comprend:

- les bases de données historiques (feux et informations météorologiques des dix dernières années) ;

- les programmes de calcul de l'indice Forêt-Météo de la méthode canadienne d'évaluation des dangers d'incendies de forêts ;

- les modèles de prévision des feux de causes humaines et de foudre;

Figure 2 RÉSEAU DE COMMUNICATIONS INFORMATISÉES EN PROTECTION CONTRE LE FEU AU QUÉBEC

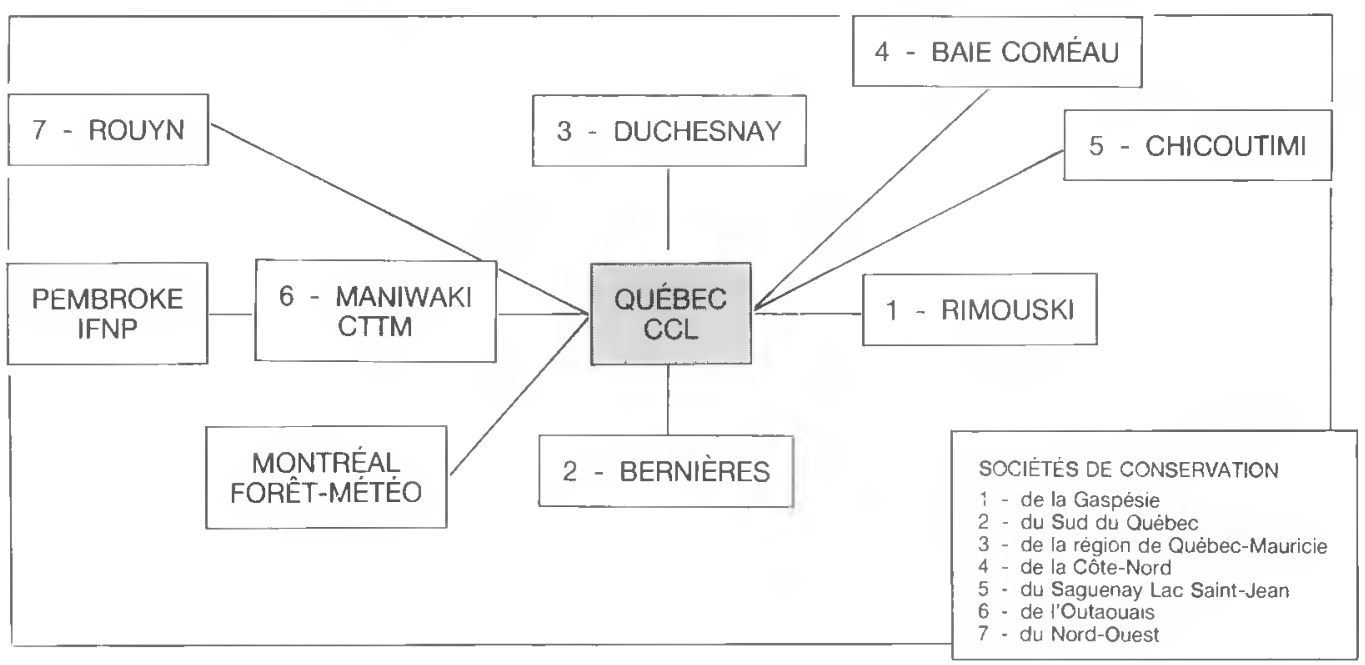


- les logiciels permettant d'effectuer les synthèses régionales et provinciales des informations et leur présentation graphique;

- le service de courrier électronique.

Certains programmes, comme les modèles de prévision des feux, ont été élaborés en fonction des conditions propres à une région du Québec. Certes, quelques années seront nécessaires pour les ajuster au contexte des autres régions.

Grâce à des recherches menées en collaboration avec l'institut forestier national de Petawawa, d'autres technologies de pointe viendront bientôt s'ajouter au Système de Gestion en Protection des Forêts.

Ainsi, on met présentement au point un réseau de radars de précipitations qui recueillera les données sur les quantités de pluie accumulées sur chaque parcelle de territoire de $2 \mathrm{~km}$ par $2 \mathrm{~km}$.

On est aussi à développer un détecteur infrarouge répondant aux besoins particuliers de la détection aérienne des débuts d'incendies forestiers atteignant par exemple un quart ou un demi-hectare et qui sont difficiles à déceler par temps de faible visibilité.

Enfin, on cherche à améliorer le modèle informatisè de propagation des incendies, qui fait appel aux données déjà intégrées au Système, dont celles relatives aux types de peuplements (par exemple les données numériques provenant d'un inventaire forestier ou d'un satellite tel le Landsat) ainsi qu'à la topographie (courbes de niveau de terrain).

\section{CONCLUSIONS}

La décision d'implanter le Système de Gestion en Protection des Forêts a constitué un élément de planification stratégique. Une fois la décision prise, il fallait être prêt à consacrer l'effort requis pour susciter l'adhésion des membres de l'organisation au Système. II fallait aussi en gérer l'implantation. Trois facteurs importants maximisent les chances de réussite dans ce domaine: le recrutement des experts requis pour assurer le fonctionnement du système, la formation des personnes qui sont mises en contact avec le SGPF et son amélioration par des évaluations périodiques. Il est prévu qu'à moyen terme, le SGPF augmentera le degré de professionnalisme et permettra à l'organisation de se positionner afin de continuer de fournir un service efficace, avec moins de ressources et en utilisant davantage les habiletés disponibles.

\section{R. PROULX}

Ingénieur forestier

Chef du Service de la Protection contre les feux

MINISTĖRE DE L'ÉNERGIE ET DES RESSOURCES

$5700,4^{e}$ avenue Ouest

Édifice de l'Atrium

CHARLESBOURG

QUÉBEC G1H 6R1 


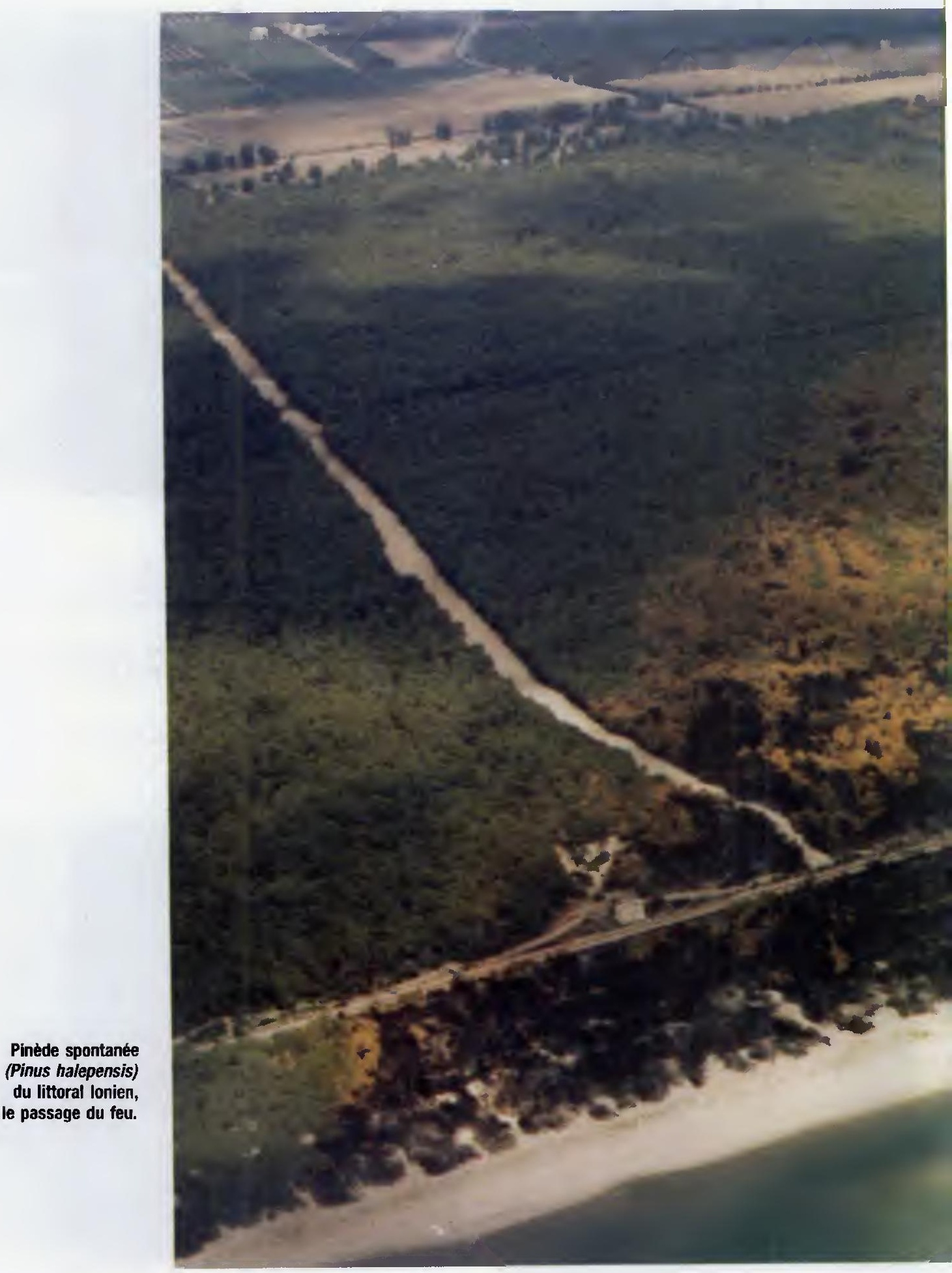




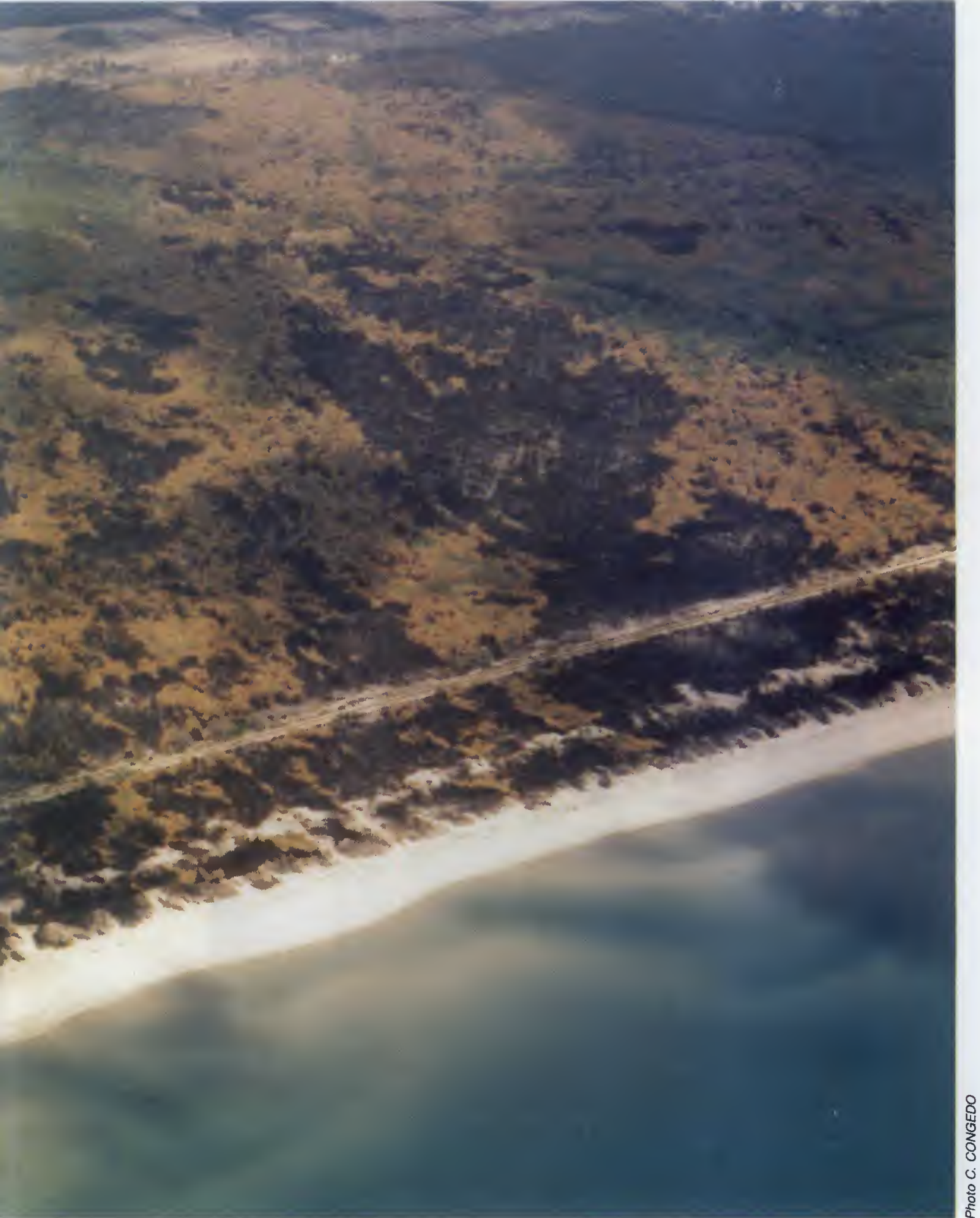




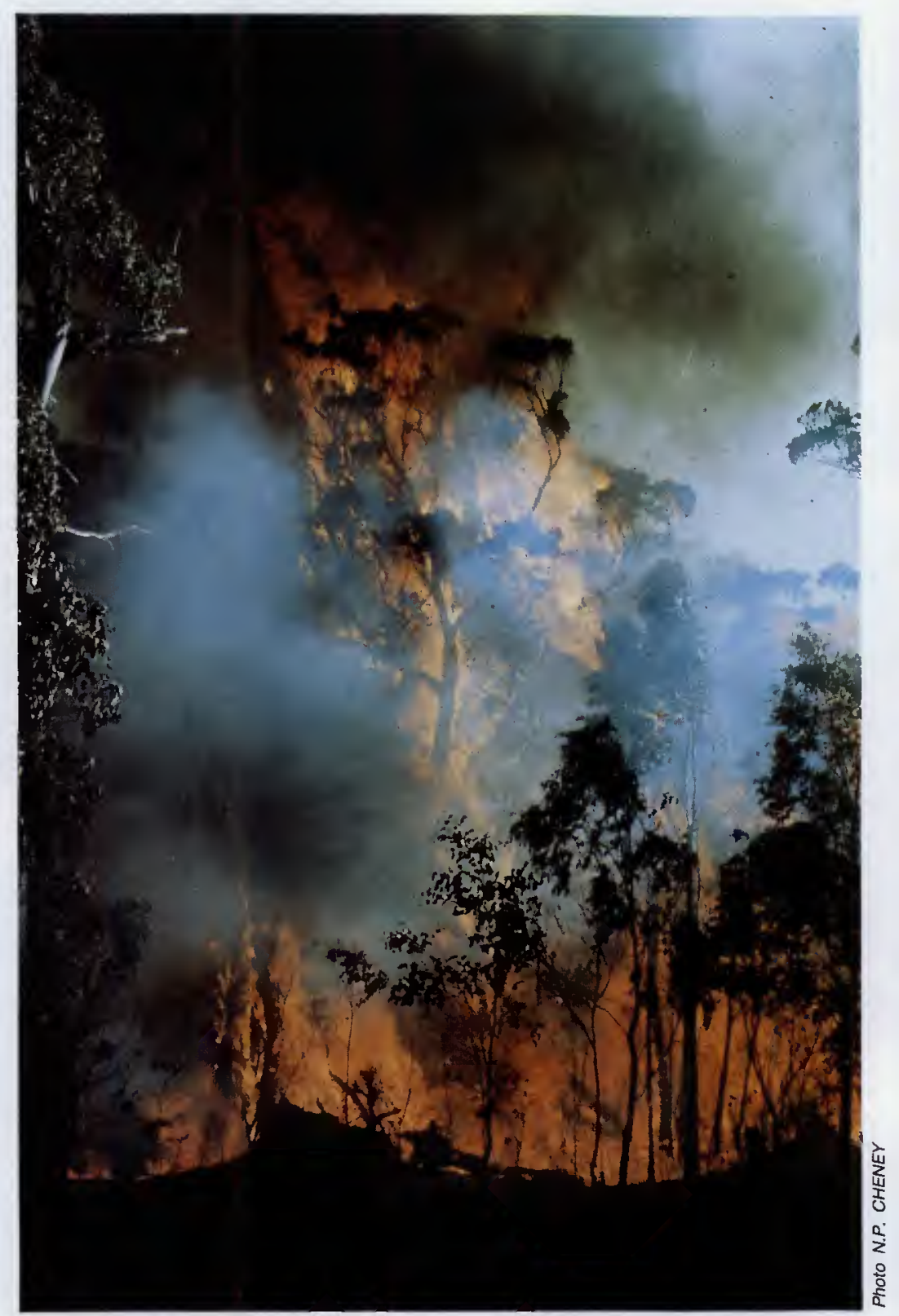

Feu des cimes dans une forêt d'Eucalyptus en Australie. 